\title{
Effect of hydrogen on surface texturing and crystallization of a-Si:H thin film irradiated by excimer laser
}

\author{
Hongliang Wang • Fernando Lusquiños • \\ Y. Lawrence Yao
}

Received: 21 February 2012 / Accepted: 22 February 2012 / Published online: 14 March 2012

(C) Springer-Verlag 2012

\begin{abstract}
Hydrogenated amorphous silicon (a-Si:H) thin films have been considered for use in solar cell applications because of their significantly reduced cost compared to crystalline bulk silicon. However, their overall efficiency and stability are lower than that of their bulk crystalline counterpart. Limited work has been performed on simultaneously solving the efficiency and stability issues of a-Si:H. Previous work has shown that surface texturing and crystallization on a-Si:H thin film can be achieved through a single-step laser processing, which can potentially alleviate the disadvantages of a-Si:H in solar cell applications. In this study, hydrogenated and dehydrogenated amorphous silicon thin films deposited on glass substrates were irradiated by $\mathrm{KrF}$ excimer laser pulses and the effect of hydrogen on surface morphologies and microstructures is discussed. Sharp spikes are focused only on hydrogenated films, and the large-grained and fine-grained regions caused by two crystallization processes are also induced by presence of hydrogen. Enhanced light absorptance is observed due to light trapping based on surface geometry changes of a-Si:H films, while the formation of a mixture of nanocrystalline silicon
\end{abstract}

\footnotetext{
H. Wang $(\bowtie) \cdot$ Y.L. Yao

Department of Mechanical Engineering, Columbia University,

New York, NY 10027, USA

e-mail: hw2288@columbia.edu

Fax: +1-212-6662393

Y.L. Yao

e-mail: yly1@columbia.edu

Fax: +1-212-8543304

F. Lusquiños

Department of Applied Physics, University of Vigo, Vigo 36310,

Spain

e-mail: flusqui@uvigo.es

Fax: +34-986-812201
}

and original amorphous silicon after crystallization suggests that the overall material stability can potentially improve. The relationship between crystallinity, fluence and number of pulses is also investigated. Furthermore, a step-by-step crystallization process is introduced to prevent the hydrogen from diffusing out in order to reduce the defect density, and the relationship between residue hydrogen concentration, fluence and step width is discussed. Finally, the combined effects show that the single-step process of surface texturing and step-by-step crystallization induced by excimer laser processing are promising for a-Si:H thin-film solar cell applications.

\section{Introduction}

Many industrial solar cells in use today use bulk materials as absorbers with crystalline silicon being the most prevalent. However, crystalline silicon suffers from the disadvantage of high material cost since relatively large thicknesses are required primarily due to its low absorption coefficient. Recently, thin film absorbers are becoming more attractive based on their potential for low-cost modules, possibility to create tandem junctions and large-scale manufacturability [1-3]. a-Si:H is the most popular material for use in thin film form due to its low energy economy (cost/watt). The main issue with a-Si:H is the high order of dangling bonds which act as recombination centers that severely reduce the carrier lifetime which results in the solar cell efficiency being below $10 \%$. Additionally, this initial efficiency will decrease by $50 \%$ or more when exposed to sunlight over a period of months which is known as the Staebler-Wronski effect, or SWE [4, 5].

Due to their low efficiency and instability, thin-film a$\mathrm{Si}: \mathrm{H}$ solar cells require a highly efficient light-trapping design to absorb a significant fraction of the incident sunlight 
and techniques to increase stability against the SWE. Antireflection (AR) coatings and front-side texturing through the use of alkaline-based solutions such as $\mathrm{KOH}$ and $\mathrm{NaOH}$ etching have been used on crystalline silicon solar cells, and pulsed laser irradiation has been used to enhance light trapping on both amorphous and crystalline materials [6-9]. However, AR coating requires additional material and anisotropic wet chemical etching is not applicable for amorphous materials or thin films. In addition, in order to reduce the SWE, hybrid a-Si/nc-Si (nanocrystalline silicon) tandem modules have been developed and are able to achieve both higher efficiency and stability compared with single-junction a-Si:H [10] due to the use of a thinner a-Si layer and the wider spectral absorption of nc-Si. To eliminate the need for two separate deposition steps which are required to form these tandem cells, laser-induced crystallization of a-Si:H has been proposed to produce a mixture of nc-Si:H and a-Si:H and simultaneously form a light trapping texture on the surface of the material [9]. Therefore, laser-based treatment of a-Si:H may solve its efficiency and stability issues in a one-step process, which is a promising methodology for thin-film solar cell fabrication.

Extensive work on surface texturing of crystalline bulk silicon had been reported by using both femtosecond and nanosecond laser [11, 12]. Wang et al. [13, 14] and Nayak and Gupta [9] also achieved both surface texturing and crystallization on a-Si:H thin films by using femtosecond laser. Compared to the femtosecond laser, nanosecond laser can induce more crystallinity due to the longer pulse duration and more thermal effect, which can potentially improve the stability. Therefore, the feasibility of nanosecond laserinduced surface texturing and crystallization of a-Si:H becomes of interest. Many research groups focused on excimer laser induced crystallization on non- or low-hydrogen a-Si thin films [15, 16], and Mathe et al. [17] reported the effect of hydrogen on surface roughness and crystallization of a$\mathrm{Si}$ :H thin films by single-pulse irradiation of excimer laser. However, there is no work showing a sharp spiked surface on the a-Si:H thin film after multiple excimer laser pulses irradiation, and how hydrogen plays a role in the laser-induced surface texturing and crystallization is still not clear.

In this paper, the formation of a densely packed, spiked surface structure has been studied through scanning electron microscopy (SEM) and atomic force microscopy (AFM) for excimer laser irradiation of a-Si:H thin films, and light absorptance dependence of surface structure is studied by spectrophotometry. The time-resolved analysis of melting and solidification of a-Si:H sample was analyzed by in situ front-side transient reflectance measurement. The effect of laser processing on crystallinity and microstructure is investigated by X-ray diffractometry (XRD) and transmission electron microscopy (TEM), and the hydrogen concentration is measured by Fourier transform infrared spectroscopy
(FTIR). The experimental results show that the hydrogen is necessary for fabricating sharp spikes on a-Si:H thin films, and the combined effects of light trapping by surface structuring and crystallization illustrate a one-step process for potentially enhancing the efficiency and stability of a-Si:H thin-film solar cells.

\section{Background}

2.1 Hydrogen effect on nanosecond laser-induced surface texturing on a-Si:H

Mathe et al. [17] showed that the surface roughness caused by single-pulse irradiation on a-Si:H could be almost two orders of magnitude larger than that of non-hydrogenated a-Si, and mentioned that the movement of $\mathrm{H}_{2}$ gas bubbles generated by the combination of $\mathrm{H}$ atoms and molecules in the ambient medium is responsible for the surface roughness. Since there is no literature showing sharp spikes formed on a-Si:H surface by multiple nanosecond pulses, the mechanism of the spike formation on a-Si:H is likely the combination of surface texturing on non-hydrogenated Si and hydrogen movement which improves the texturing behavior. Therefore, it is necessary to understand the formation mechanism of surface texturing on non-hydrogenated Si before studying that on a-Si:H.

Nanosecond laser can produce micro-conical/column spikes on bulk crystalline silicon (c-Si) surface at the fluence of $1-5 \mathrm{~J} / \mathrm{cm}^{2}$ after a few hundred to thousands of pulses. The mechanism can be divided into two steps: spike initialization and growth. Dehghanpour et al. [18] indicated that the sharp spikes are initially caused by the generation of capillary waves due to the micro-fluid mechanics of the molten layer, and then the periodic bead-like structure is formed as the seed of the final spikes. The development of the spikes is explained based on the formation of preferential removal of the material through vapor-liquid-solid (VLS) mechanism [12]. The silicon-rich vapor generated between the spikes preferentially redeposits on the molten tip of the microcolumns due to high accommodation coefficient of liquid, while the vapor prefers to dissolve into the liquid rather than solid, so that those spikes grow uniformly with the increasing of laser pulses. At the sides of the columns, because of the increased reflectivity with increasing incident angle, they are not melted or ablated.

\subsection{Hydrogen effect on crystallization of a-Si:H}

Partial crystallization of a-Si:H is helpful to improve the stability, and larger grains with less grain boundaries are more desirable microstructures since defects and impurities are always located at grain boundaries. Hydrogen may have 
some drawbacks on the crystallization effect. First, hydrogen could trigger explosive crystallization [15]. When the incident laser energy density is only sufficient to induce partial melting of a-Si layer, explosive crystallization can be observed and it converts a-Si into fine-grained polycrystalline Si via a melt-meditated mechanism [19]. The buried liquid layer is at a temperature higher than the melting point of a$\mathrm{Si}$ but lower than the melting point of $\mathrm{c}-\mathrm{Si}$, and propagates into the a-Si layer by simultaneously melting of a-Si and solidifying into c-Si. Due to latent heat difference between $\mathrm{c}-\mathrm{Si}$ and a-Si, the propagation is self-sustaining. Two different nucleation scenarios-a moving liquid/solid Si interface can be unstable with respect to nucleation of c-Si [20] and small crystallites can nucleate in a-Si during heating phase prior to melting [21] — had been made to explain why explosive crystallization occurs. Second, an observation by Mathe et al. [17] showed that hydrogen slows down the crystallization process because more energy density is needed to crystallize a given depth when hydrogen is present. Largegrained polycrystallites are generated at the hydrogen depletion region near the surface, and fine-grained ones are located at hydrogen contained area underneath.

Therefore, hydrogen could cause fine-grained polycrystallites by explosive crystallization and spend part of the laser energy that slows down the crystallization process. However, these effects may be alleviated by multiple-pulse radiation which will be discussed later.

\section{Experimental setup}

Amorphous silicon films were deposited on 0.525-mm-thick Corning 1747 glass substrates using plasma enhanced chemical vapor deposition (PECVD). The a-Si:H film was deposited at a rate of $60 \AA / \mathrm{s}$ in an hydrogen diluted silane environment at $380{ }^{\circ} \mathrm{C}$ with a hydrogen atomic concentration of around 20 at $\%$. Film thickness was found to be roughly $1.6 \mu \mathrm{m}$ through ellipsometry measurements [13]. The a-Si:H films were cleaned with acetone in an ultrasonic cleaner for 5 minutes and then rinsed with methanol and distilled water prior to processing.

Surface texturing and crystallization were carried out using an unpolarized $\mathrm{KrF}$ excimer laser with a wavelength of $248 \mathrm{~nm}$ and pulse duration of $30 \mathrm{~ns}$. After passing through an attenuator, the laser beam is homogenized by a multi-lens homogenizer system and then further shaped as it passes through a photomask. This shape is then demagnified $(5 \times)$ and focused onto the sample through a projection lens. This results in a homogeneous $1 \mathrm{~mm} \times 1 \mathrm{~mm}$ square irradiated spot at the sample surface. The samples were mounted on a three-axis translation stage and irradiated by one to a hundred laser pulses with various fluence values $(0.15$ to $0.5 \mathrm{~J} / \mathrm{cm}^{2}$ ) at a stationary spot, and moved to an adjacent point for the next irradiation until an area of 10 by $10 \mathrm{~mm}$ is treated. For the step-by-step process, the condition starts at $0.05 \mathrm{~J} / \mathrm{cm}^{2}$ and ends at the desired final laser fluence $(0.3$ and $0.4 \mathrm{~J} / \mathrm{cm}^{2}$ ) using step widths from 0.01 to $0.08 \mathrm{~J} / \mathrm{cm}^{2}$ and 50 or 100 pulses at each step.

The untreated and treated samples were observed through SEM. Surface roughness and the distribution of spikes in the treated samples were also examined using an AFM. Crystallinity and material microstructure after laser irradiation are analyzed by XRD and TEM, and the hydrogen concentration is investigated by FTIR. The time-resolved analysis of melting and solidification of a-Si:H sample was analyzed by in situ front-side transient reflectance measurement. The optical transmittance and reflectance of asreceived and laser-treated a-Si:H films were measured by a spectrophotometer over a wavelength range of 250-2500 $\mathrm{nm}$ which corresponds to the main spectral range of solar irradiation [22]. The reflectance ( $R$ in \%) and transmittance $(T$ in \%) are then used to calculate the absorptance ( $A$ in \%) of the film: $A=100-R-T$.

\section{Results and discussion}

\subsection{Effect of hydrogen on surface morphology}

\subsubsection{Surface topography on laser irradiated a-Si:H}

Lowndes et al. [12] showed that in the polarized pulsed-laser irradiation on $\mathrm{c}-\mathrm{Si}$, two different phenomena may be seen: at lower fluence $\left(<1 \mathrm{~J} / \mathrm{cm}^{2}\right)$, laser-induced periodic surface structure (LIPSS) occurs on the silicon surface; at higher fluence (1 to $5 \mathrm{~J} / \mathrm{cm}^{2}$ ), columnar or conical spikes of a few tens of micrometers in height are generated. However, in texturing a-Si:H thin films, a much lower and narrower processing window is tested in order to not damage or crystallize the entire film. In this study, the amorphous samples are irradiated at stationary locations with lower fluence $\left(0.15\right.$ to $\left.0.5 \mathrm{~J} / \mathrm{cm}^{2}\right)$ and number of pulses (1 to 100). A typical result achieved using a fluence of $0.4 \mathrm{~J} / \mathrm{cm}^{2}$ is shown in Fig. 1. The average spike height and spacing after 1 pulse measured by AFM [13] are $460.8 \pm 10.6 \mathrm{~nm}$ and $1.637 \pm 0.037 \mu \mathrm{m}$, respectively, where the variation represents standard deviation. As discussed in Sect. 2.1, beyond the capillary waves formed by solidifying of the molten layer, hydrogen should play an important role in formation of the spikes. It is showed that the hydrogen atoms combine to form $\mathrm{H}_{2}$ gas, and then diffuse into the ambient medium and leave the blister regions after laser processing [17]. However, a more complex process could occur, i.e., the combined hydrogen molecules may explode when the laser energy is high enough; subsequently, the released pressure drives the upper liquid silicon layer to move upwards while the surrounding liquid silicon flows to the explosion position. The liquid silicon solidifies before it achieves equilibrium, which forms much 

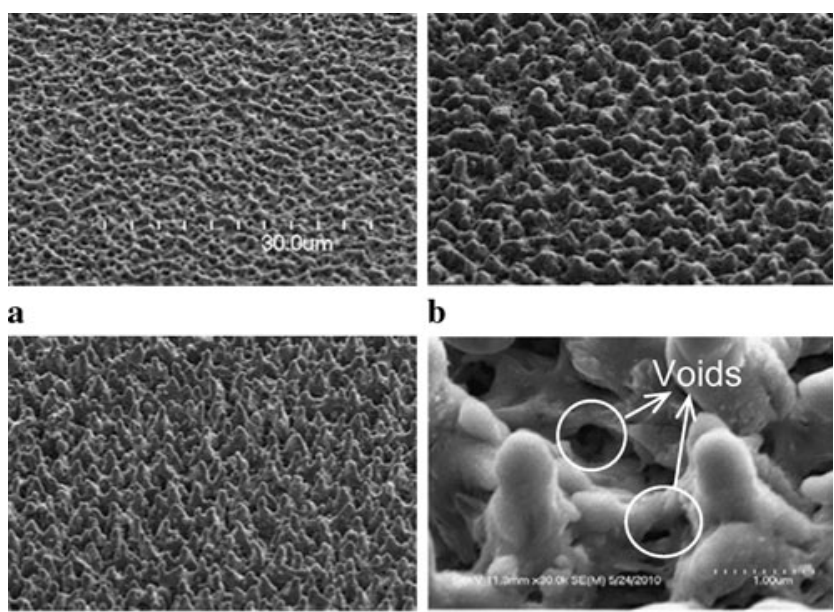

c

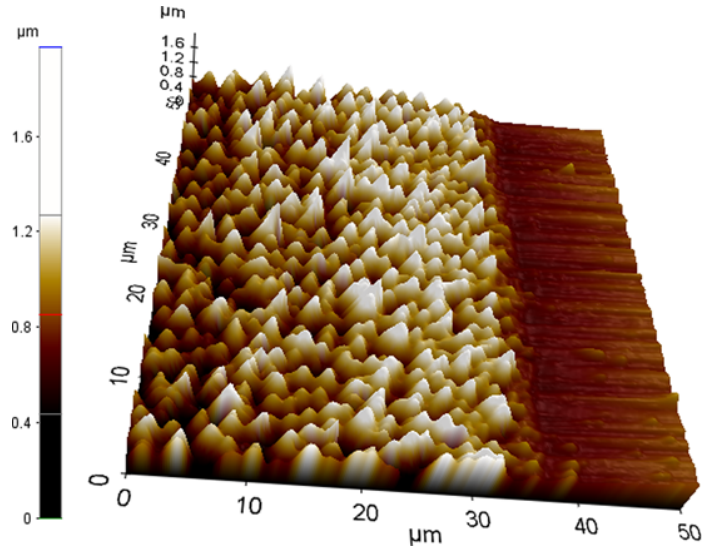

Fig. 2 AFM image of surface morphology at the laser irradiation boundary of a-Si:H film at a fluence of $0.4 \mathrm{~J} / \mathrm{cm}^{2}$ with 50 pulses, showing the spikes are protruded from the original surface

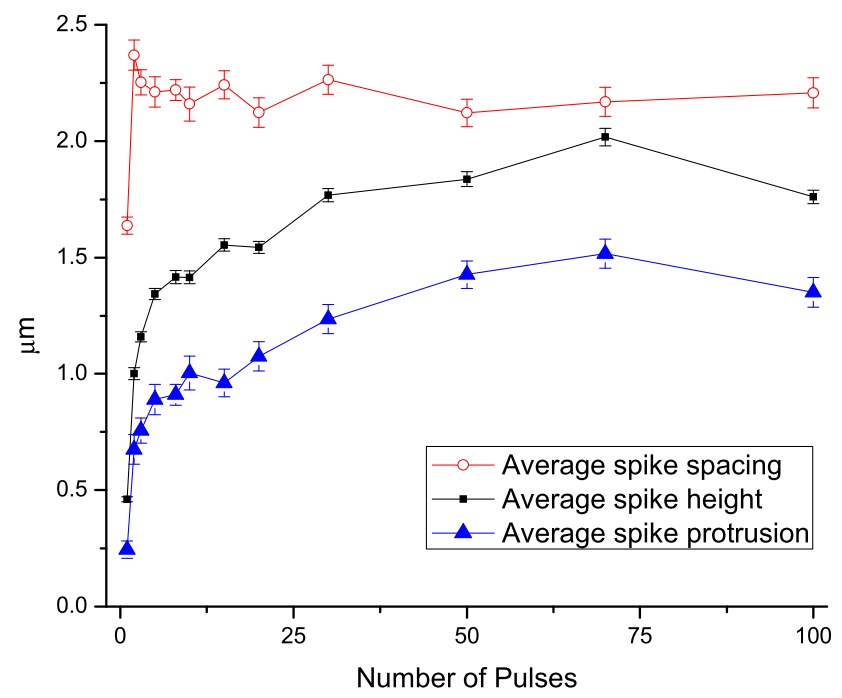

Fig. 3 Dependence of average spike height, protrusion and spacing on number of pulses for a fixed fluence of $0.4 \mathrm{~J} / \mathrm{cm}^{2}$, determined from an area of $50 \times 50 \mu \mathrm{m}^{2}$ at the processing center; error bars indicate standard deviation

for the first 10 pulses or so, the spike height and protrusion are increased rapidly due to both effects of hydrogen explosion and VLS caused by multiple reflection in between the spikes; second, from 10 pulses to about 70 pulses, the hydrogen molecules are mostly depleted so that the VLS mechanism is dominant, and therefore the average spike height increase rate slows down; third, after 70 pulses, the average spike height increases to around $2 \mu \mathrm{m}$, and the silicon-rich ablated material in between the spikes is difficult to reach to the tips so that VLS cannot happen. As a result, the average spike height and protrusion start decreasing. The average spacing remains a constant of around $2.2 \mu \mathrm{m}$ after the first pulse. This is because the molten layer solidifies before reaching equilibrium at the first pulse and forms a higher tightened surface structure due to the presence of hydrogen, 


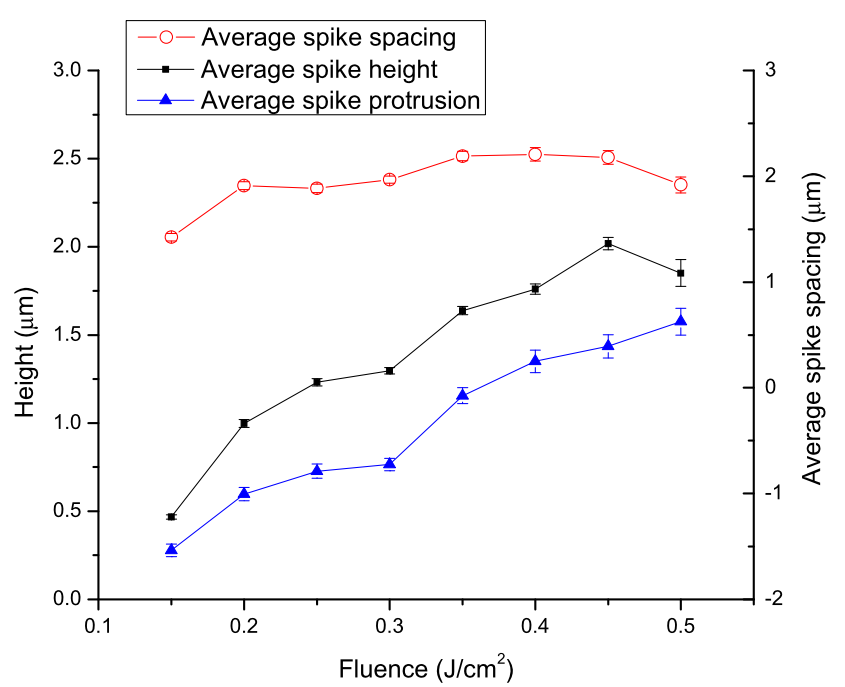

Fig. 4 Dependence of average spike height, protrusion and spacing on laser fluence for a fixed number of pulses (100 pulses), determined from an area of $50 \times 50 \mu \mathrm{m}^{2}$ at the processing center; error bars indicate standard deviation

so that the remelting layer by the second pulse cannot be stable. The liquid silicon will be redistributed due to the surface tension effect and is stabilized until the spike bases are connected together before solidification. After that, the spikes spacing already initially formed cannot be affected by subsequent pulses. The average spike height, protrusion and spacing dependent on different fluence values at 100 pulses are investigated in Fig. 4. It shows that the average spike spacing remains the same under different fluence values and the average spike height and protrusion increase with the increasing of the fluence until it reaches around $2 \mu \mathrm{m}$. As discussed above, when the spikes are high enough, VLS mechanism is not dominant so that the spike height starts decreasing, that is why the average spike height becomes lower at $0.5 \mathrm{~J} / \mathrm{cm}^{2}$ than that of $0.45 \mathrm{~J} / \mathrm{cm}^{2}$. However, the spike protrusion still remains the highest at $0.5 \mathrm{~J} / \mathrm{cm}^{2}$, which indicates that the depth between the undamaged material and original surface is lower. The reason is that more material can be ablated under a higher fluence; however, when the spike height is high enough, the ablated material cannot reach the tips of the spikes. Therefore, the material could only be redeposited back to the valley between the spikes which cause a shallower depth. The average spike spacing is around $1.6 \mu \mathrm{m}$ at $0.15 \mathrm{~J} / \mathrm{cm}^{2}$ and remains between 2 and $2.2 \mu \mathrm{m}$ at higher fluence, which may be due to the surface stabilization during the initial spike base formation. The exception at $0.15 \mathrm{~J} / \mathrm{cm}^{2}$ is because no spike is formed at this point and the surface structure is formed more tightened similarly to the first pulse effect.
Table 1 List of a-Si properties used in the simulation calculation

\begin{tabular}{llll}
\hline a-Si property & Units & Value & Literature source \\
\hline$\rho$ & $\mathrm{g} / \mathrm{cm}^{3}$ & 2.2 & {$[24]$} \\
$C_{p}$ & $\mathrm{~J} / \mathrm{gK}^{-1}$ & 0.952 & {$[24]$} \\
$K$ & $\mathrm{~W} / \mathrm{m}^{-1} \mathrm{~K}^{-1}$ & 0.007 & {$[24]$} \\
$R$ & & 0.54 & {$[25]$} \\
$\eta$ & $\mathrm{mPa} \mathrm{s}^{-1}$ & $0.7-0.8$ & {$[26]$} \\
$T_{m}$ & $\mathrm{~K}$ & 1423 & {$[24]$} \\
$T_{v}$ & $\mathrm{~K}$ & 3538 & {$[24]$} \\
$\partial \sigma / \partial T$ & $\mathrm{~N} / \mathrm{mK}^{-1}$ & $7.4 \times 10^{-5}$ & {$[26]$} \\
$\alpha$ & $\mathrm{cm}{ }^{-1}$ & $1.5 \times 10^{6}$ & {$[27,28]$} \\
$L_{f}$ & $\mathrm{Cal} / \mathrm{g}$ & 315 & {$[27]$} \\
$\sigma$ & $\mathrm{mN} / \mathrm{m}$ & 850 & {$[26]$} \\
\hline
\end{tabular}

\subsubsection{Surface topography on dehydrogenated a-Si}

In order to study the effect of hydrogen on surface morphology, a numerical simulation and experiments of spike formation on laser irradiated dehydrogenated a-Si are investigated to compare with that of a-Si:H samples.

For the first pulse, the heat transfer phenomenon can be obtained by Eq. (1) [23]:

$\rho(T) C_{P}(T) \frac{\partial T(\vec{r}, t)}{\partial t}-\nabla[\kappa(T) \nabla(T(\vec{r}, t))]=0$

where $\rho, C_{P}, \kappa, T, r$ and $t$ are density, specific heat, thermal conductivity, temperature, radius and time, respectively. The properties of a-Si used in the simulation are listed in Table 1.

The geometry of the irradiated sample can be considered as semi-infinite, because the thermal penetration depth (Eq. (2)) is lower than the thickness of the sample [29]:

$L_{T}=2 \sqrt{\delta \tau_{l}}$

where $\delta$ is the thermal diffusivity and $\tau_{l}$ is the laser pulse duration.

Assuming the sample material is isotropic and uniform, the laser radiation is approximately uniform and the transversal dimension of the irradiated area is larger than the thermal penetration depth, the problem can be considered as a unidimensional model and the temperature field will depend exclusively on the depth ( $z$-axis) [30]. The variation of the thermo-physical parameters $\left(\rho, C_{P}, \kappa\right)$ of the a-Si film with the temperature is not big enough to be considered, and average values of these parameters are taken into account. The convection and radiation losses at temperatures between 1000 and $2000 \mathrm{~K}$ hardly exceed $1-3 \mathrm{~W} / \mathrm{cm}^{2}$ [27], so that they are negligible during the simulation.

With these hypotheses, Eq. (1) with the initial and boundary conditions can be written as follows:

$\rho C_{P} \frac{\partial T(z, t)}{\partial t}-\kappa \nabla^{2}[T(z, t)]=0$ 


$$
\begin{aligned}
& T(z, t=0)=T_{\text {ambient }} \\
& \kappa\left[\frac{\partial T(z, t)}{\partial z}\right]_{z=0}=Q_{\text {laser }}-Q_{\text {ambient }}-Q_{\text {radiation }} \\
& \kappa\left[\frac{\partial T(z, t)}{\partial z}\right]_{z=\infty}=0
\end{aligned}
$$

where $Q_{\text {laser }}=I(x, y, t)(1-R) q(t)$, and $I(x, y, t)$ is the laser intensity, $R$ is the reflectance and $q(t)$ is the temporal dependence of the laser pulse.

The thermal gradient in the molten pool drives the motion of the liquid silicon provoking the deformation of the surface, and the pressure differences created at a curved interface support the evolution of the deformations on the liquid surface, which is known as capillary waves with the dispersion relationship between the wave-vector $k$ and the angular frequency $\omega$ given by [26]:

$\omega^{2}=\frac{\alpha k^{3}}{\rho} \tanh (k h)$

where $\alpha$ is the surface tension of the liquid and $h$ is the depth of the molten pool.

In order to obtain the amplitude of the thermo-capillary waves, the Navier-Stokes equation has been solved for a stationary and incompressible fluid stated by Eq. (8) with the boundary conditions of Eqs. (9) and (10):

$\nabla \vec{v}=0, \nabla^{2} \vec{v}=0$

$\vec{v}=0 \quad$ at $z=-h$

$\eta\left(\frac{\partial v_{r}}{\partial z}+\frac{\partial v_{z}}{\partial r}\right)=\frac{\partial \alpha}{\partial r}=\frac{\partial \alpha}{\partial T} \frac{\partial T}{\partial r} \quad$ at $z=0$

where $\vec{v}$ is fluid velocity, $\eta$ is the dynamic viscosity, and the subscripts $r$ and $z$ label the radial and normal components of the velocity, respectively.

Equation (8) is integrated in $z$-direction [31] that provides the change in the morphology of the fluid $\Delta_{z}$ due to the motion of the wave:

$\Delta z=-\frac{1}{2 \eta r} \frac{\partial \alpha}{\partial T} \frac{\partial}{\partial r}\left[r\left\langle h^{2}\right\rangle \frac{\partial I}{\partial r}\right]$

with $I=\int_{T_{S}>T_{m}}\left(T_{S}-T_{m}\right) d t$ and $\left\langle h^{2}\right\rangle I=\int_{T_{S}>T_{m}} h^{2}\left(T_{S}-\right.$ $\left.T_{m}\right) d t, T_{s}$ the surface temperature and $T_{m}$ the melting temperature of a-Si.

For multiple pulses, the following hypotheses are established: first, the influence of the phase transformation after the pulses on the thermal and optical properties is negligible; second, the reflectance is only affected by the change of surface roughness. The simulated spike heights at different fluence and number of pulses are shown in Fig. 5. The spike height at 1 or 100 pulses almost linearly increase with increasing of fluence. According to reference [17], the spike

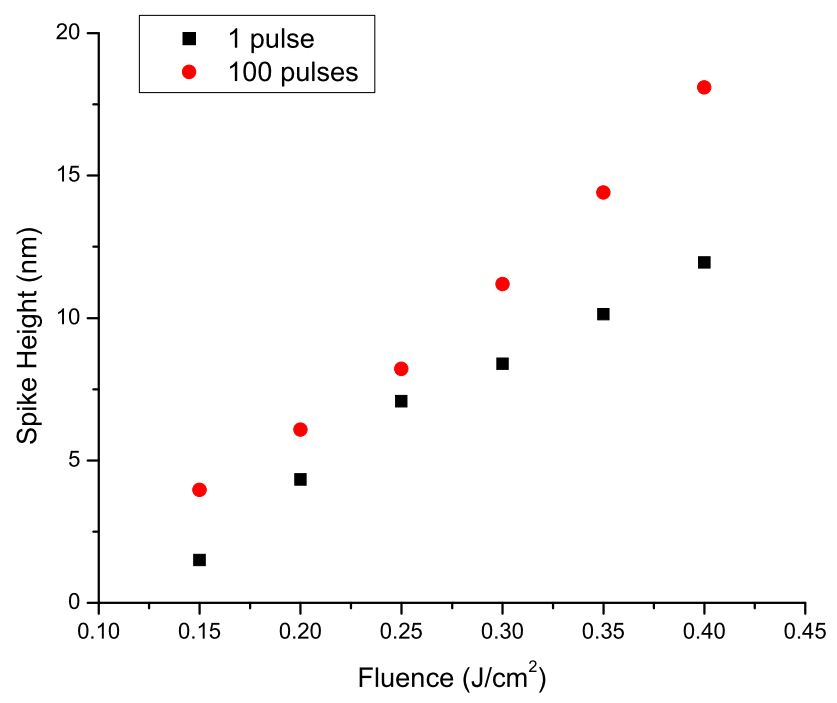

Fig. 5 Simulation results of dependence of the spike height on laser fluence for a fixed number of pulses (1 and 100 pulses)

heights at 1 pulse are close to the experimental results. The average spike height of laser irradiated dehydrogenated a$\mathrm{Si}$ thin films (prepared by thermal annealing at 80 hours) at $0.4 \mathrm{~J} / \mathrm{cm}^{2}$ and 100 pulses is around $58 \mathrm{~nm}$. Although this is in the same order of magnitude as the simulation result, the numerical predictions underestimated the spike height. The reason could firstly be that the reflectance is not a constant spatially, i.e. the light absorption inside the valleys is more than that in the peaks. Therefore, more material is actually ablated and redeposited on the tips. Secondly, the material in the simulation is uniform, but in reality, a-Si has defects and impurities, such as residual hydrogen, and these imperfect properties could intensify the light absorption and increase the spike height.

In order to show the effect of hydrogen on surface morphology experimentally, the a-Si:H samples are annealed in a vacuum furnace at $450{ }^{\circ} \mathrm{C}$ [32] for different amounts of time to diffuse out hydrogen as much as possible. Figure 6 shows the FTIR spectra measured for different annealing time. The non-hydrogen a-Si sample (100-nm-thick a-Si on $\mathrm{SiO}_{2}$-coated glass) is introduced as a reference spectrum. The hydrogen concentration can be estimated from peaks caused by the stretching mode of $\mathrm{Si}-\mathrm{H}$ at $2000 \mathrm{~cm}^{-1}$ and $\mathrm{SiH}_{2}$ at $2100 \mathrm{~cm}^{-1}$ using equation [32]:

$N=A \int \frac{\alpha}{\omega} d \omega=A \frac{S}{t \omega_{0}}$

where $N, A, \alpha, \omega, t, \omega_{0}$, and $S$ are the hydrogen density, the conversion factor $\left(9 \times 10^{9} \mathrm{~cm}^{-2}\right.$ at $2000 \mathrm{~cm}^{-1}$ and $2.2 \times$ $10^{10} \mathrm{~cm}^{-2}$ at $2100 \mathrm{~cm}^{-1}$ ), the absorbance, the frequency, the film thickness, the peak frequency, and the peak area, respectively.

Based on Eq. (12), the initial $\mathrm{H}$ concentration of $16.07 \%$ is significantly reduced to $1.71 \%$ after thermal annealing of 
Fig. 6 Transmission infrared spectra for thermal annealed a-Si:H samples at different annealing time, showing hydrogen concentration decreases with increasing of annealing time

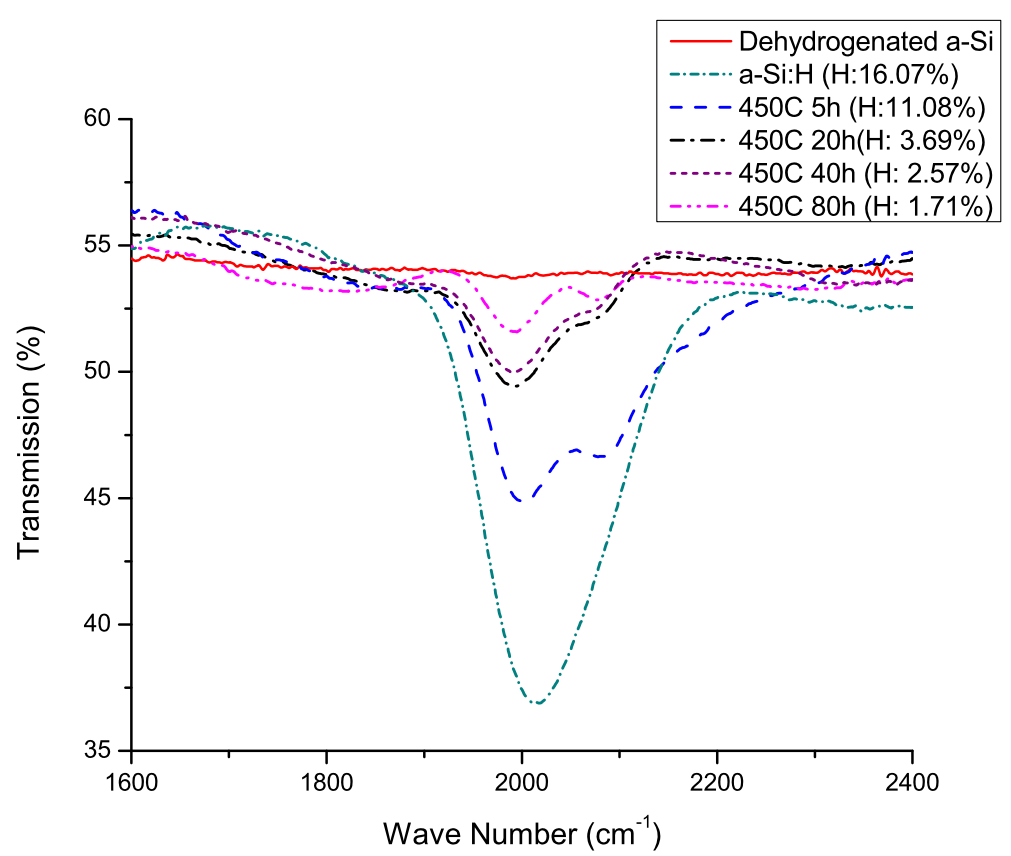

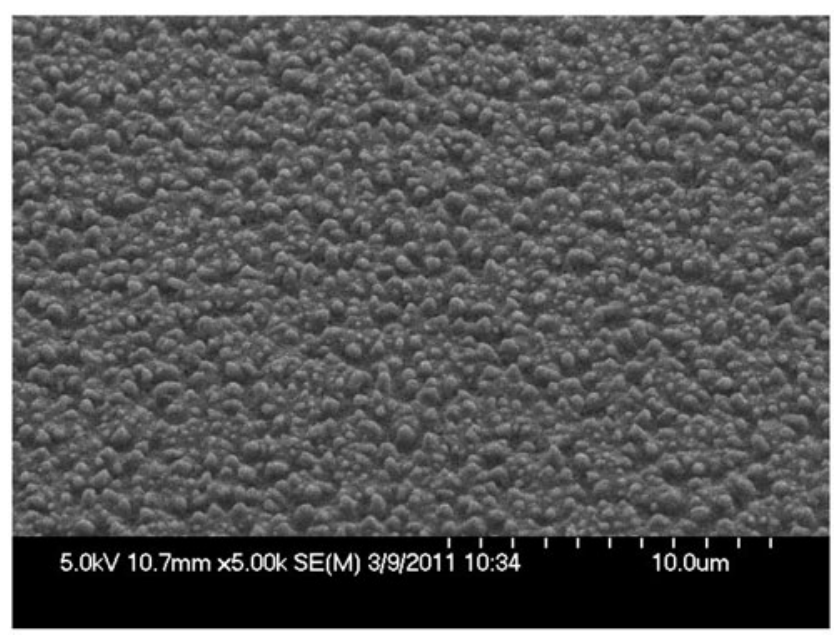

Fig. 7 SEM image of 80-hour thermal annealed a-Si:H film surface after being irradiated at a fluence of $0.4 \mathrm{~J} / \mathrm{cm}^{2}$ and 100 pulses, showing no sharp spikes on the surface

80 hours, and the XRD measurement shows that the thermal annealed sample still remains amorphous (not shown). The SEM image of laser-processed 80-hour-annealed sample at a fluence of $0.4 \mathrm{~J} / \mathrm{cm}^{2}$ with 100 pulses is shown in Fig. 7, and the average spike height is $57.8 \pm 2.15 \mathrm{~nm}$. It can be seen that no sharp spikes are formed without the presence of hydrogen. As discussed above, the molten layer can form capillary waves after solidification; however, the amplitude of the wave is too small to introduce the light multiple reflection towards the valleys. Therefore, when the next pulse comes, the irradiated area will be melted again similarly as the first pulse does and no spikes are formed after multiple pulses. This is different from the femtosecond laser experi- ments, where ablation is dominant and the sharp spikes can be fabricated due to the different ablation rate when the periodic surface structure is formed. Moreover, dehydrogenate a-Si thin films cannot stand the higher fluence used for c-Si, since it is easy to damage the entire film. Therefore, from both the simulation and experiment results, the hydrogen is necessary for forming sharp spikes on a-Si:H thin films by a nanosecond laser.

\subsection{Effect of hydrogen on crystallization and absorptance}

In order to study the crystallization of the treated films, $\mathrm{X}$-ray diffraction patterns taken for the untreated and treated a-Si:H films are shown in Fig. 8. The spectra have been shifted vertically for clarity. All the spectra show an amorphous peak around $2 \theta=25^{\circ}$, which is caused by the internal constructive interference of the amorphous $\mathrm{Si}$ and glass network. Three peaks at around $2 \theta=29^{\circ}, 47^{\circ}$ and $56^{\circ}$ emerge after processing which indicate a structural change after laser irradiation and can be indexed to the (111), (220) and (311) crystalline orientations of silicon. The normalized crystallinity is estimated by the calculated area differences of the measured spectra between the laser-processed and untreated samples. The largest area at the condition of $0.4 \mathrm{~J} / \mathrm{cm}^{2}$ and 100 pulses is defined as a crystallinity of $100 \%$, and the other conditions are calculated by dividing it. The normalized crystallinity at conditions of $0.4 \mathrm{~J} / \mathrm{cm}^{2}$ with 50 pulses and $0.3 \mathrm{~J} / \mathrm{cm}^{2}$ with 100 pulses are 98.98 and $57.38 \%$, respectively. It is understood that under partial melting regime, the higher the fluence, the higher the crystallinity. However, under the same fluence, when the number of pulses achieves a certain number, the crystallinity levels 
Fig. 8 X-ray diffraction spectra of as-received a-Si:H film, laser treated films at $0.4 \mathrm{~J} / \mathrm{cm}^{2}$ with 50 and 100 pulses, and $0.3 \mathrm{~J} / \mathrm{cm}^{2}$ with 100 pulses.

There is no signs for crystallinity for the untreated sample and are three different peaks for (111), (220) and (311) orientation of silicon for all laser treated samples. The spectra have been shifted for clarity
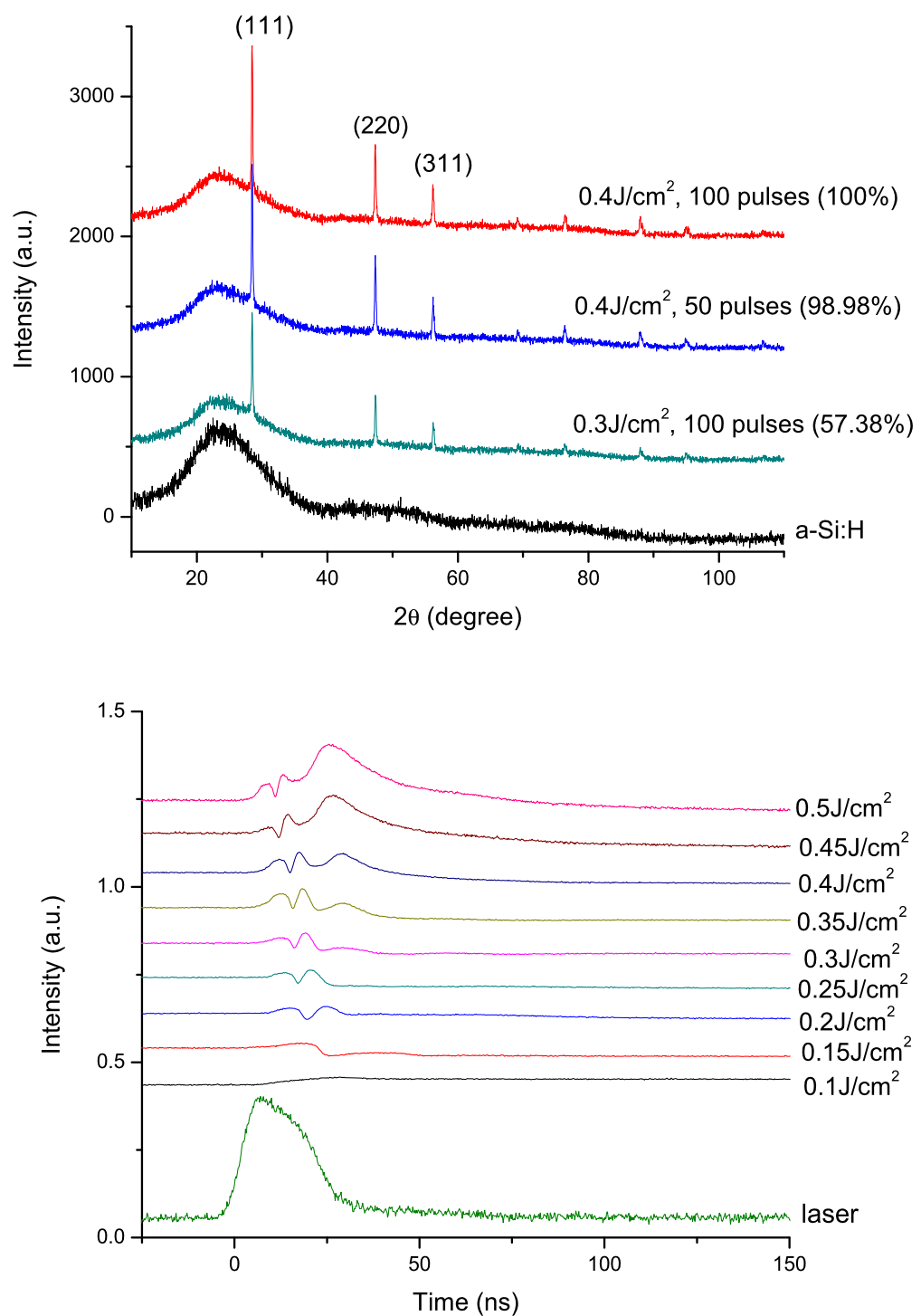

Fig. 9 The in situ front-side transient reflectance signals of a-Si:H films irradiated at different fluence values off. This is because the spikes can reach a certain height to avoid the ablated material redepositing to the tips, so that no more amorphous material can be melted underneath the crystallized layer.

\subsubsection{Hydrogen effect on crystallization}

Single-pulse laser irradiation as well as in situ front-side transient reflectance (FTR) measurement is performed for analyzing the dynamics of melting and solidification of a$\mathrm{Si}: \mathrm{H}$ thin films. Figure 9 shows the FTR signals correlation with different fluence values $\left(0.1\right.$ to $\left.0.5 \mathrm{~J} / \mathrm{cm}^{2}\right)$. It can be seen that no damage or phase changes at $0.1 \mathrm{~J} / \mathrm{cm}^{2}$. The signal intensity decrease at $0.15 \mathrm{~J} / \mathrm{cm}^{2}$ indicates that the material starts being crystallized since the reflectivity of $\mathrm{c}-\mathrm{Si}$ is lower than that of a-Si. When the fluence is higher than $0.15 \mathrm{~J} / \mathrm{cm}^{2}$, the signal oscillation, due to the interference effect resulting from the fast-moving melting buried layer, shows that explosive crystallization occurs [15] at the beginning. The following plateau means that the solidified surface has been remelted by the rest of the pulse, and it can be seen that higher fluence always has a longer lifetime of the molten pool, which may cause larger grains after crystallization. $\mathrm{Hu}$ [33] showed that no explosive crystallization occurs for excimer laser irradiated dehydrogenated a-Si deposited by PECVD. Therefore, hydrogen must play a role for triggering explosive crystallization. However, the reason is not clear at this point. It is suggested that the $\mathrm{H}$ atom moving may absorb or release the energy and change the temperature in the local area. At some point, nucleation could occur due to the heating or change of the molten Si cooling rate locally, and these nuclei will be the seeds triggering the explosive crystallization.

Figure 10 shows the cross-sectional TEM images at the fluence of $0.4 \mathrm{~J} / \mathrm{cm}^{2}$ with different number of pulses. It can be seen that after the first pulse, microstructure of the mate- 


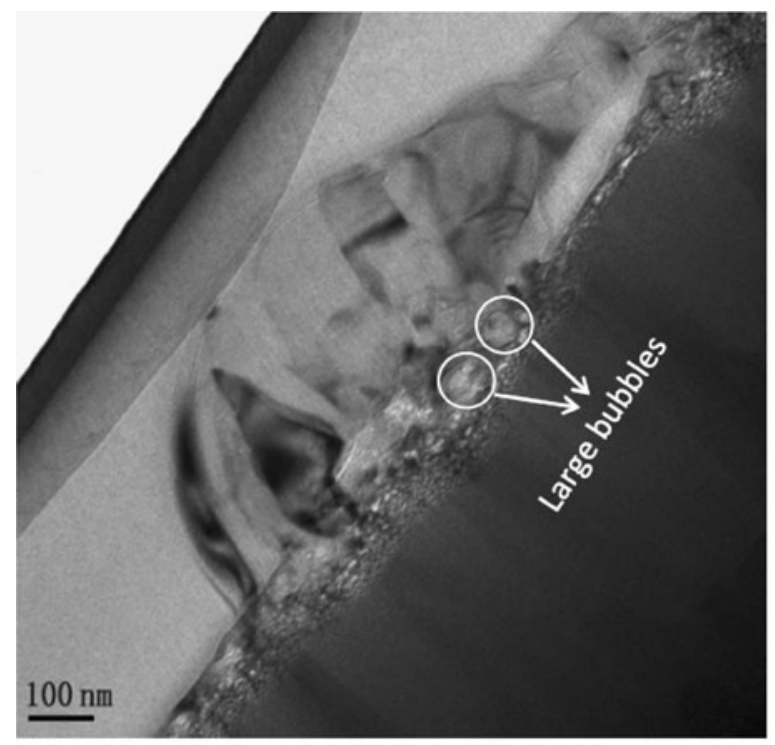

a

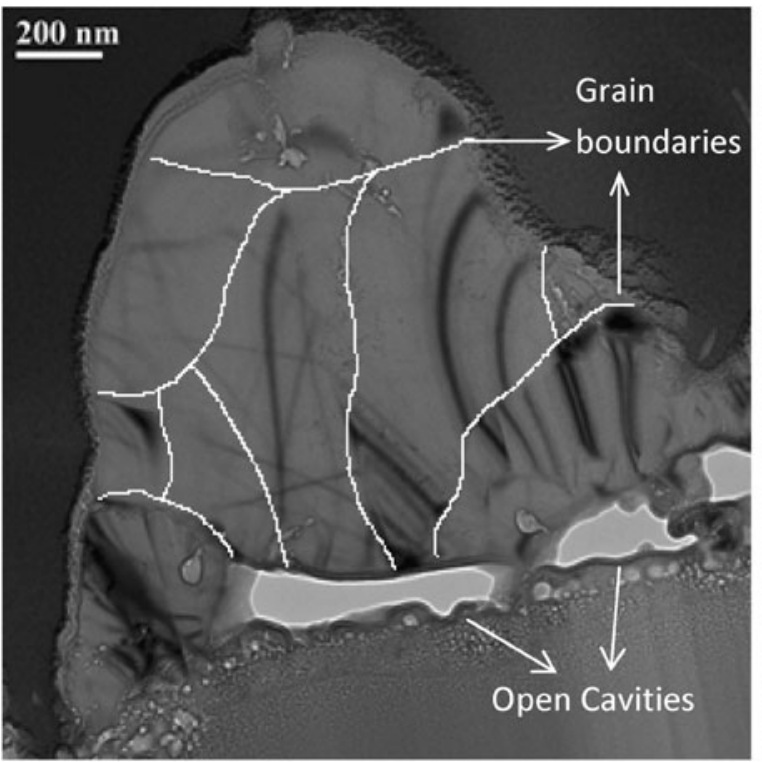

c

50 pulses

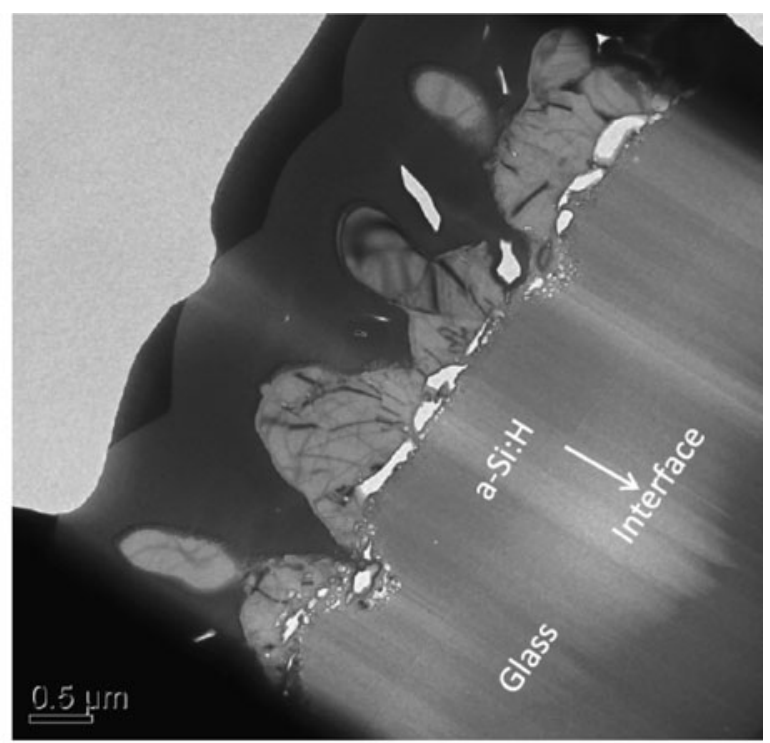

b

50 pulse

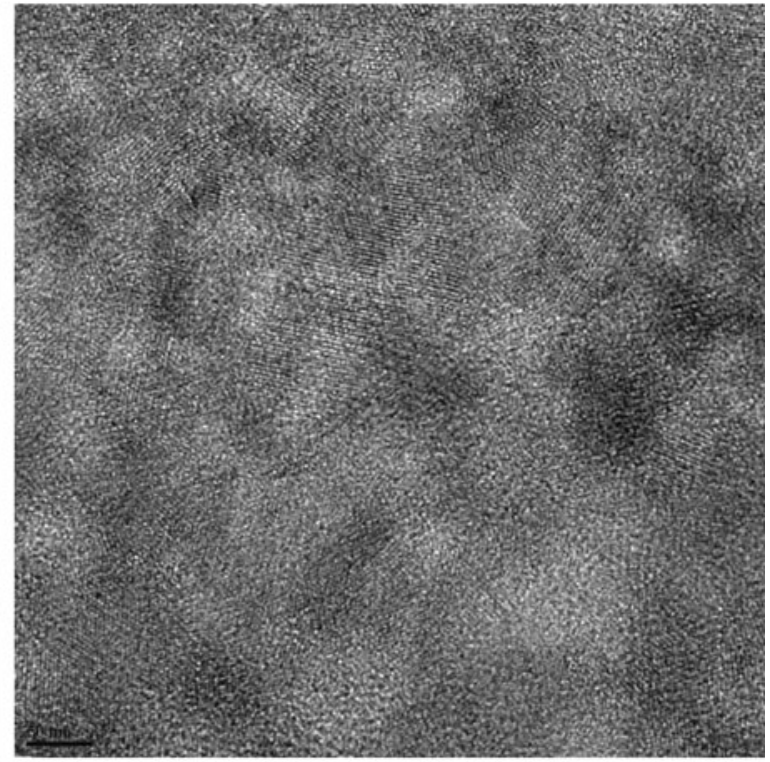

d

Fig. 10 Cross-sectional view TEM micrographs of a-Si:H samples irradiated at (a) 1 and 50 pulses at (b) low, (c) high magnification and (d) high-resolution TEM image with a fixed fluence of $0.4 \mathrm{~J} / \mathrm{cm}^{2}$

rial can be divided into two regions-large- and fine-grained regions. The FTR signal shows that explosive crystallization occurs within $20 \mathrm{~ns}$ which forms the fine-grained region, and after that, the top layer has been melted again because of the heat accumulation by the rest of the pulse. The hydrogen atoms inside the material start moving by absorbing the laser energy and aggregating at the interface between the solid and liquid silicon. Those hydrogen atoms combine as molecules and form as bubbles, and those bubbles keep dilating and explode when the internal pressure inside the bubbles becomes higher than the outside. The bubble explosion at the liquid/solid silicon interface drives the liquid silicon to move up and the surrounding liquid flows towards it. Before the liquid silicon reaches equilibrium, the material has solidified and formed as spikes with a height of $\sim 350 \mathrm{~nm}$ and left large bubbles at the interface as shown in Fig. 10(a). Since the molten layer at the surface can last for almost $20 \mathrm{~ns}$, the crystallized material can form much larger grains of $\sim 140 \mathrm{~nm}$ than that of explosive crystallization. Figure 10(b) shows the cross-sectional TEM image of a-Si:H sample irradiated at 50 pulses under low magnification. It can be seen that the spike height and spacing are around 1.5 and $2 \mu \mathrm{m}$, respectively. Since TEM samples cannot be prepared to be exactly at the center of the spike, this is why the spike height 
measured by TEM is always lower than that measured by AFM, but the spike spacing measurements are similar. The connected open cavities underneath the spikes are caused by the hydrogen bubble explosions. Since the melting temperature of c-Si is around $225 \mathrm{~K}$ higher than that of a-Si [19] and a broad disorder zone exists at the interface between the large- and fine-grained regions after first pulse, the excited hydrogen atoms during multiple-pulse processing will preferentially move to this interface. Therefore, more and more bubbles explode at the same location and form connected open cavities. In order to estimate the grain size after 50 pulses, one of the spikes is focused during TEM measurement and the sample was tilted with a step of $0.1^{\circ}$ in both $x$ and $y$ directions to avoid the effect of band contours. Since different grains will have different band contours at a certain direction, the moving region of the same contour shows the grain region. As shown in Fig. 10(c), the largegrained region is only located on the spike and the largest grain size is around $400 \mathrm{~nm}$. Figure 10(d) shows the highresolution TEM image taken at the interface of large- and fine-grained regions, and the grain sizes are around $40 \mathrm{~nm}$. When moving even deeper to the substrate, the grain size becomes smaller and smaller until reaching the amorphous region. This can also prove that the presence of explosive crystallization after the first pulse, since the buried liquid layer of explosive crystallization is only a-few-nanometers thick and very small grains are formed, and those tiny bubbles are formed by hydrogen effect during the explosive crystallization process.

\subsubsection{Hydrogen effect of crystallization dependent on pulse duration and pulse number}

The effect of hydrogen during the laser processing depends on the pulse duration. As shown in Fig. 11, the a-Si:H sample is irradiated by a femtosecond laser with a pulse duration of $130 \mathrm{fs}$ and a condition of $0.4 \mathrm{~J} / \mathrm{cm}^{2}$ at a scan speed of 1 $\mathrm{mm} / \mathrm{s}$. Much less bubbles and void area are observed from the image, which may be caused by a different crystallization process induced by femtosecond laser. Wang et al. [14] show that a non-thermal melting occurs within a picosecond and material starts being ablated or thermally vaporized afterwards due to the energy transfer back from electrons to the material, at last, a very thin layer underneath can be thermally melted and crystallized after solidification. Therefore, the thermal effect which can cause hydrogen explosion inside the material is only a less dominant process, so that hydrogen effect is much less during femtosecond laser process. Another difference between the two different laser processes is that the large-grained layer is covered over a conical spike with fine grains on an amorphous matrix. This is because the ablation is dominant formation mechanism by the femtosecond laser. When the spikes are formed after a certain number

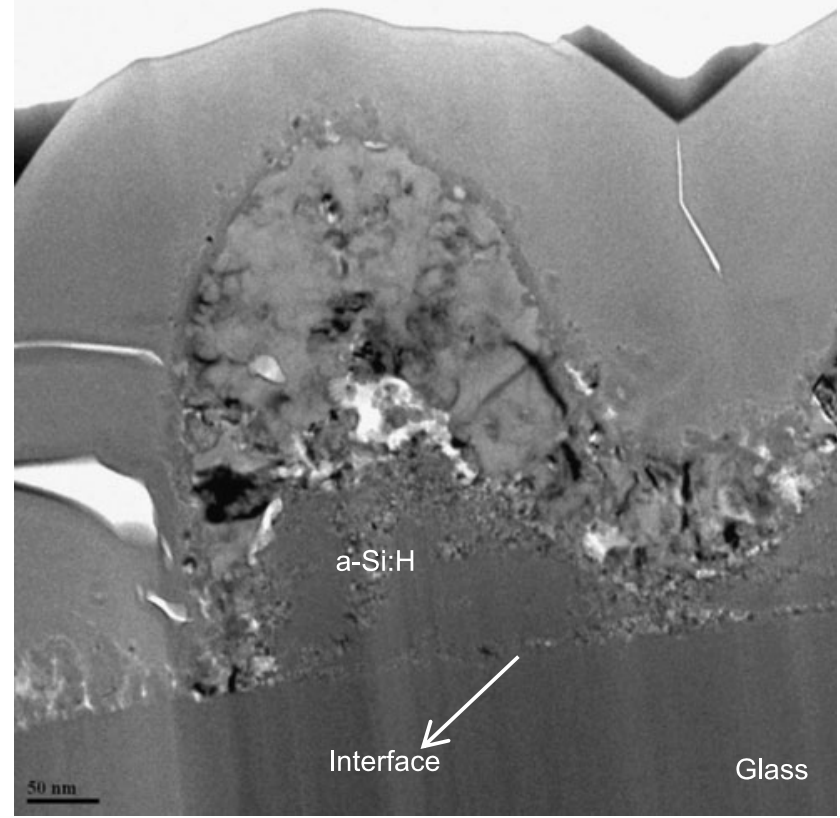

Fig. 11 Cross-sectional view TEM micrograph of a-Si:H samples irradiated by 130 -fs laser pulses at a fluence of $0.4 \mathrm{~J} / \mathrm{cm}^{2}$ and a scan speed of $1 \mathrm{~mm} / \mathrm{s}$

of pulses, the crystallized top layer on the spikes will protect the underneath material from being ablated, and the ablated material around the spikes will deposit preferentially to the tips to form a thicker and thicker crystallized layer. Compared to Fig. 10(c), much more defects are observed in the large-grained region in femtosecond laser irradiated a-Si:H films, which could act as a combination center of the photoexcited electron-hole pairs, and therefore the cell efficiency and stability could be better by excimer laser processed samples rather than femtosecond laser.

Figure 12 shows the maximum grain size at a fluence of $0.4 \mathrm{~J} / \mathrm{cm}^{2}$ with different number of pulses. An observation of maximum grain is achieved at 5 pulses. For the first two pulses, due to the hydrogen effect, part of the pulse energy is absorbed by hydrogen and used for generating fine-grained crystallites caused by explosive crystallization. So the grain size increases when the number of pulses increases while the hydrogen concentration decreases. After 5 pulses, the hydrogen is almost depleted, and the spikes are formed. It is noticed that the diameter of the spikes becomes smaller and smaller due to the light multi-reflection between the spikes, and the material around the spike has been ablated, deposits on the tips and crystallizes to a random orientation. Therefore, the grain size starts decreasing since the large grains are only located at the spikes. When the spikes are sharp enough after 25 pulses, no more material can be ablated from the side of the spikes, and the grain size remains unchanged. 


\subsubsection{Hydrogen effect on absorptance}

The absorptance spectra of untreated and laser-treated samples are shown in Fig. 13. It can be seen that the absorptance increases over the entire spectrum. The absorptance spectra of the laser-processed samples under different conditions are similar due to the similar light-trapping surface geometries, and the absorptance at the below-band-gap region could be caused by the impurities and defects generated during the laser processing. It is noted that the absorptance decreases rapidly at the wavelength of $\sim 300 \mathrm{~nm}$, which may be caused by the interference at the open cavities. As shown

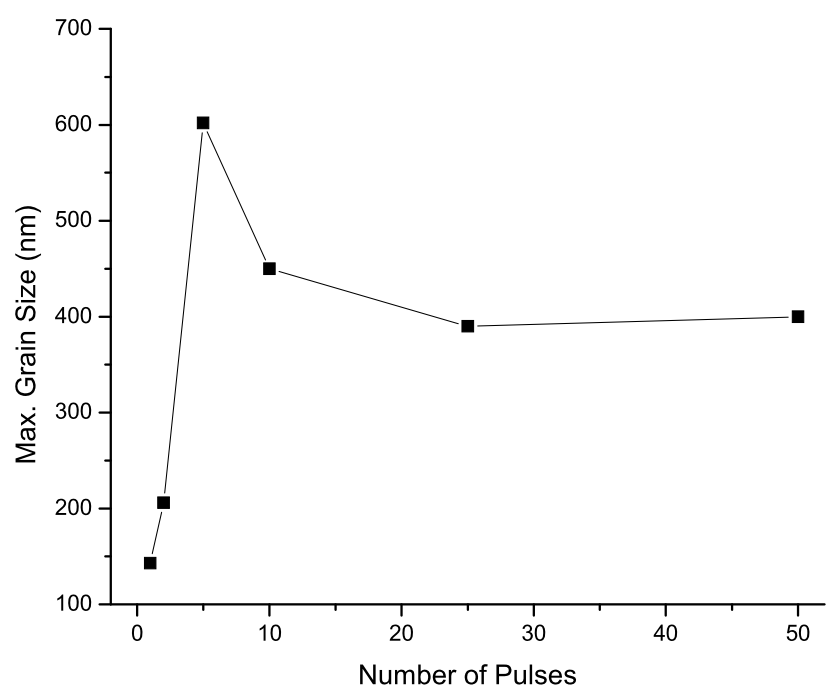

Fig. 12 Dependence of maximum grain sizes of laser-irradiated $\mathrm{a}-\mathrm{Si}: \mathrm{H}$ films on number of pulses at a fluence of $0.4 \mathrm{~J} / \mathrm{cm}^{2}$ in Fig. 10(c), the depth of the open cavity is around 150 $\mathrm{nm}$ and it is based on the constructive interference equation $2 n d=m \lambda$, where $n, d, m$ and $\lambda$ are refractive index, cavity depth, integer and wavelength. Here, $n=1$ and $d=150$ $\mathrm{nm}$, therefore the corresponding wavelengths that can introduce constructive interference for light reflection are 300, $150,100 \mathrm{~nm}$, etc. This explains why the absorptance spectra get the lowest value at around the wavelength of $300 \mathrm{~nm}$.

\subsection{Effect of step-by-step process on residual hydrogen concentration, crystallinity and absorptance}

Hydrogen is used for passivation of the defects in the Si network and grain boundaries in order to increase the efficiency of the solar cell. However, from Fig. 14, the FTIR spectra show that there is no hydrogen left after laser processing at the conditions of 0.3 and $0.4 \mathrm{~J} / \mathrm{cm}^{2}$ with 100 pulses. Therefore, leaving as much hydrogen as possible after processing is another criterion. Lengsfeld et al. [34] show that a residual hydrogen concentration of $\sim 5 \%$ was observed after stepby-step excimer laser induced crystallization of a-Si:H with an initial hydrogen content of $10 \%$.

In this study, step-by-step processes starting at a fluence of $0.05 \mathrm{~J} / \mathrm{cm}^{2}$ and ending at 0.3 and $0.4 \mathrm{~J} / \mathrm{cm}^{2}$ with increment of $0.05 \mathrm{~J} / \mathrm{cm}^{2}$ are carried out. As shown in Fig. 14, the samples with an ending fluence of $0.4 \mathrm{~J} / \mathrm{cm}^{2}$ have no hydrogen left after processing, whether 50 or 100 pulses are applied for each step. In order to understand the effect of the increment on residual hydrogen concentration, stepby-step processes with a desired final fluence of $0.3 \mathrm{~J} / \mathrm{cm}^{2}$ and different increments from 0.01 to $0.08 \mathrm{~J} / \mathrm{cm}^{2}$ are taken, and 100 pulses are used for each process sequence. It can
Fig. 13 Comparison of absorptance spectra measured by spectrophotometry of as-received and laser irradiated $\mathrm{a}-\mathrm{Si}: \mathrm{H}$ films at fluence values of $0.4 \mathrm{~J} / \mathrm{cm}^{2}$ with 50 and 100 pulses, and $0.3 \mathrm{~J} / \mathrm{cm}^{2}$ with 100 pulses

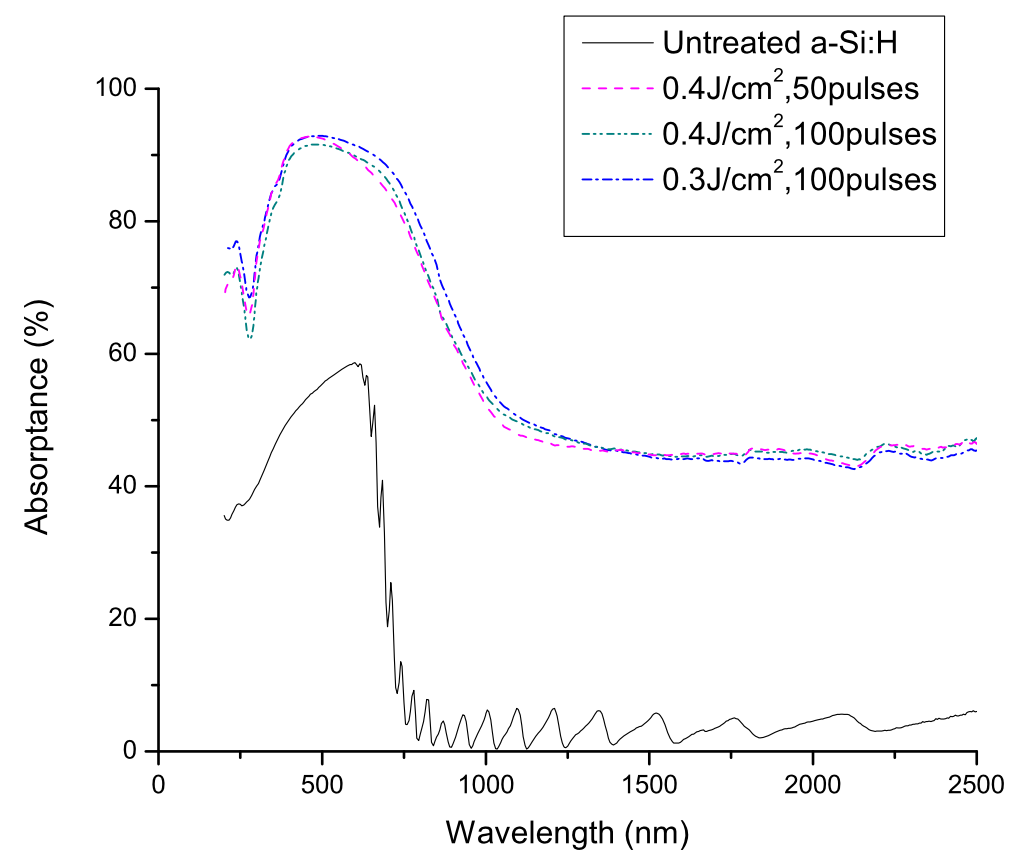



spectra for single-step and step-by-step laser-processed a-Si:H samples, where EF, SW and \# indicate ending fluence, step width and number of pulses, respectively
Fig. 14 Transmission infrared

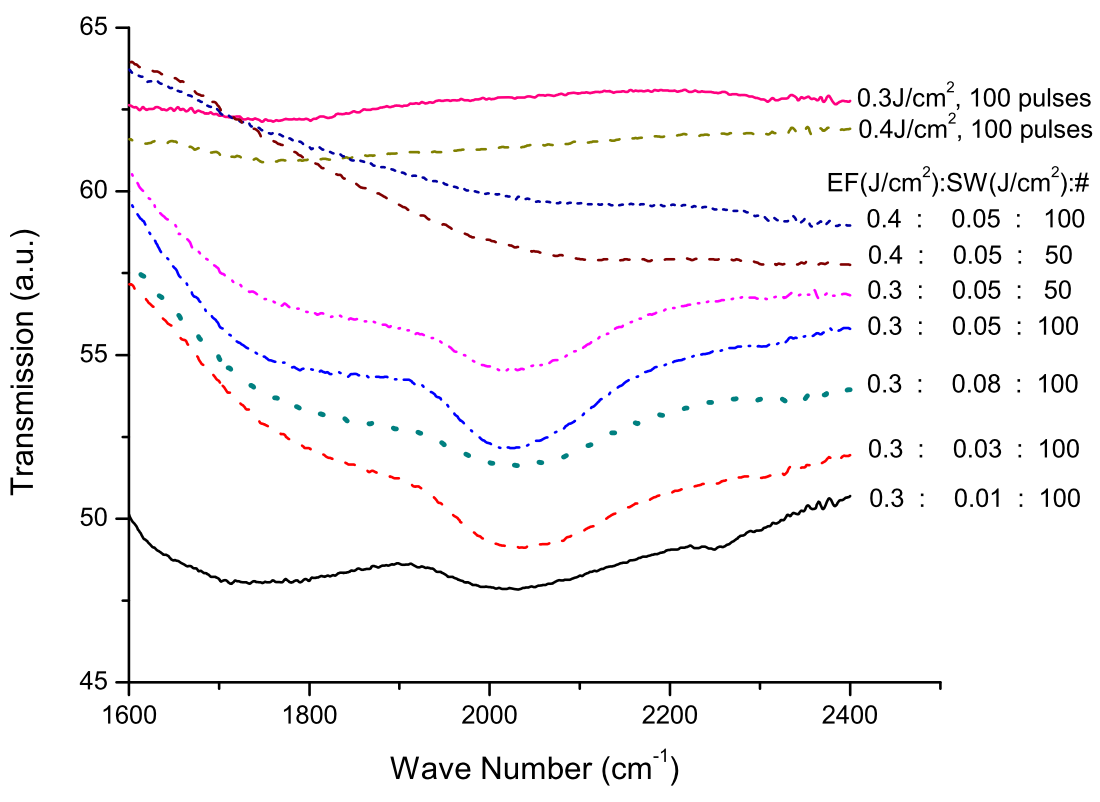

Fig. 15 Normalized crystallinity and hydrogen concentration of step-by-step laser-processed a-Si:H samples at a fixed initial fluence of $0.05 \mathrm{~J} / \mathrm{cm}^{2}$ and final fluence of $0.3 \mathrm{~J} / \mathrm{cm}^{2}$ with different increments and number of pulses

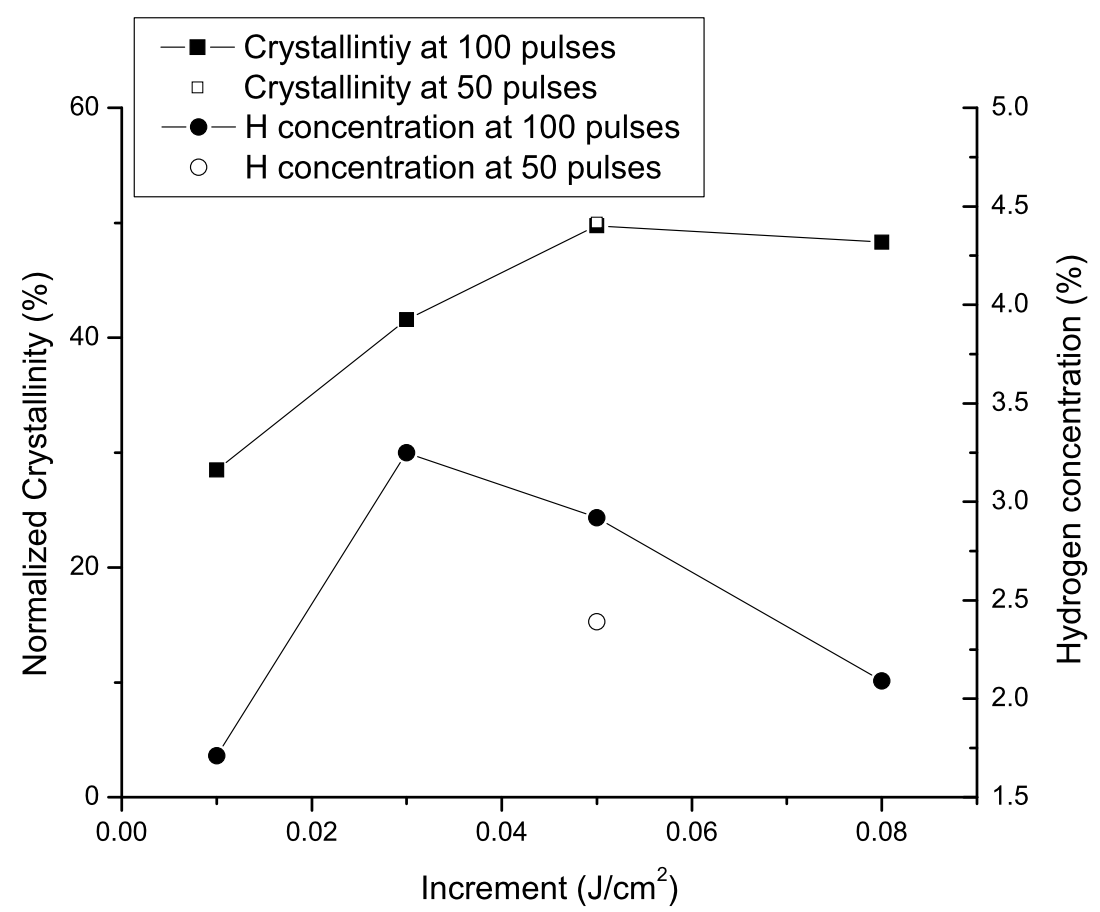

be seen that the hydrogen concentration varies with different increment. The quantitative characteristics of hydrogen concentration and normalized crystallinity based on Eq. (12) and XRD measurement are performed with the conditions that have residual hydrogen left, and the results are shown in Fig. 15. For 100 pulses at each step, a maximum hydrogen concentration is achieved at $3.25 \%$ with a step width of $0.03 \mathrm{~J} / \mathrm{cm}^{2}$. Based on the FTR measurement, the a-Si:H sample starts to be crystallized at $0.15 \mathrm{~J} / \mathrm{cm}^{2}$. At the step of $0.01 \mathrm{~J} / \mathrm{cm}^{2}$, there are almost 10 steps before the structure phase change. However, the hydrogen inside the material will be diffused out due to the laser-induced thermal effect, which is more than $450{ }^{\circ} \mathrm{C}$ shown in Fig. 6, during those undamaged steps. Therefore, the hydrogen concentration at the step of $0.01 \mathrm{~J} / \mathrm{cm}^{2}$ is smaller than that of $0.03 \mathrm{~J} / \mathrm{cm}^{2}$. When the step goes to $0.05 \mathrm{~J} / \mathrm{cm}^{2}$, thermal annealing by lower fluence is less dominant since only 2 to 3 steps before the crystallization threshold. However, the fluence at each step plays a more important role. Since the crystallized material performs as a protection layer for hydrogen from diffusing out, at the step of $0.03 \mathrm{~J} / \mathrm{cm}^{2}$, after the crystallization threshold, the laser pulse at each step is less energetic compared to that 
Fig. 16 Comparison of absorptance spectra measured by spectrophotometry of single-step and step-by-step laser-processed a-Si:H samples. The inset shows the SEM image of surface morphology of a step-by-step laser-processed a-Si:H sample with a final fluence of $0.3 \mathrm{~J} / \mathrm{cm}^{2}$, step width of $0.05 \mathrm{~J} / \mathrm{cm}^{2}$ and 100 pulses at each sequence

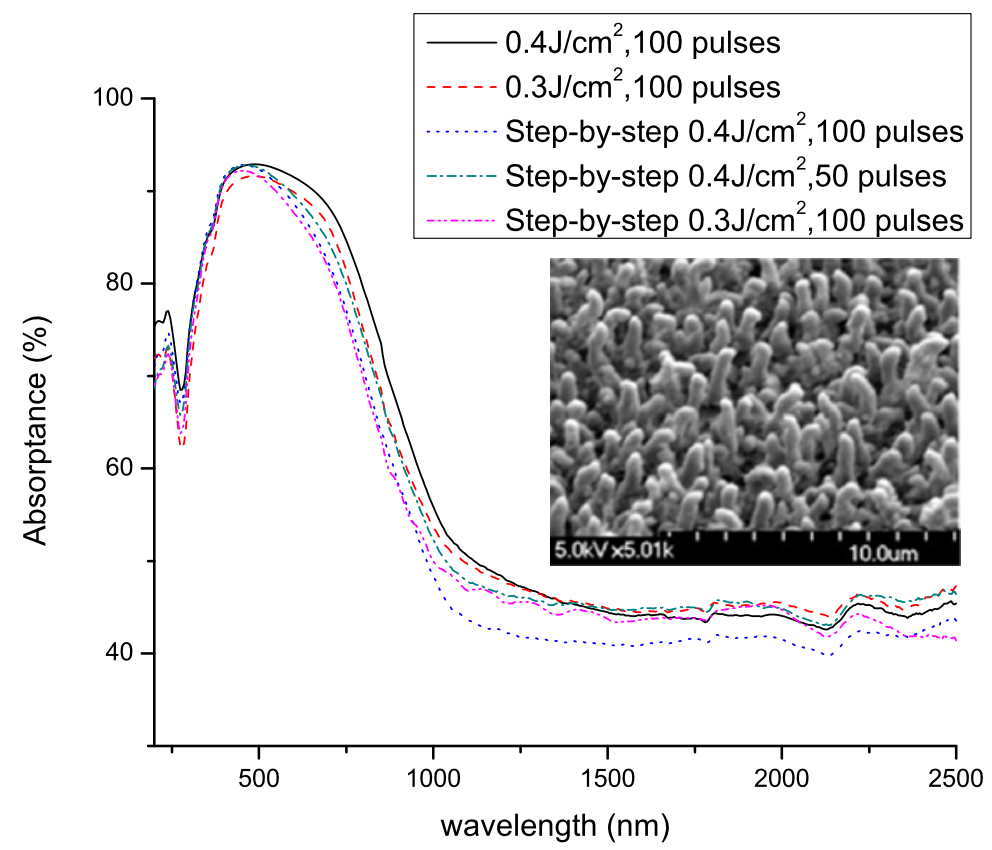

of $0.05 \mathrm{~J} / \mathrm{cm}^{2}$, which indicates that less energy is transferred underneath the crystallized layer to diffuse out the hydrogen at each step. Although more steps are taken, the final residual hydrogen is still less for the smaller-step process. Likewise, an even lower hydrogen concentration is detected at a step of $0.08 \mathrm{~J} / \mathrm{cm}^{2}$. It is also noted that, at the step of $0.05 \mathrm{~J} / \mathrm{cm}^{2}$, higher hydrogen concentration is achieved by 100 pulses at each step than that caused by 50 pulses at each step. The reason is that with the same increment, more pulses could cause a thicker crystallized layer at the crystallization threshold, and this layer can prevent too much hydrogen being diffused out from the next step. Therefore, at the same step, more pulses applied each step will retain more residual hydrogen. For crystallinity, it can be observed that crystallinity increases with increasing of increment until it reaches $0.05 \mathrm{~J} / \mathrm{cm}^{2}$ and remains at around $50 \%$ afterwards. The reason is that with a smaller step, the laser process at each step can only melt a shallower layer compared to that caused by a higher step; therefore, less material can be crystallized after each step. The final crystallinity will become in samples processed in small increment although more steps are taken. When the increment is higher than $0.05 \mathrm{~J} / \mathrm{cm}^{2}$, the crystallinity remains at around $50 \%$. This is because the spikes are grown to the saturated height (around $2 \mu \mathrm{m}$ ) at some point during the processing, and the ablated material cannot reach to the tips of the spikes. Therefore, beyond this point, only remelting and recrystallizing of the crystallized material occur at each step, but no effect on crystallinity. Likewise, at the step of $0.05 \mathrm{~J} / \mathrm{cm}^{2}$, the crystallinity is also unchanged at both 50 and 100 pulses at each step. Overall, the condition at the increment of $0.05 \mathrm{~J} / \mathrm{cm}^{2}$ and 100 pulses exhibits a relatively good performance at both cell efficiency and stability.

Figure 16 shows the comparison of absorptance between single-step and step-by-step laser-processed a-Si:H samples. It can be seen that all the laser-processed samples perform similarly. The inset shows the SEM image of a step-by-step laser-processed a-Si:H sample at a final fluence of $0.3 \mathrm{~J} / \mathrm{cm}^{2}$ with a step of $0.05 \mathrm{~J} / \mathrm{cm}^{2}$ and 100 pulses at each sequence. A similar formation of spikes is observed compared to the single-step laser-processed sample shown in Fig. 1, which explains the similar absorptance due to a similar change of surface geometry. It is also noted that the interference at around $300 \mathrm{~nm}$ is not eliminated by the step-by-step processes, which means the open cavities are still formed as shown in Fig. 10(c). Further work is in progress to avoid those cavities for improvement of the light absorption. Overall, the combined effects of surface texturing, crystallization and residual hydrogen by excimer laser induced step-by-step process (condition: a final fluence of $0.3 \mathrm{~J} / \mathrm{cm}^{2}$, a step of $0.05 \mathrm{~J} / \mathrm{cm}^{2}$ and 100 pulses at each sequence) of a-Si:H thin films yield the most desirable performance.

\section{Conclusion}

In conclusion, it has been demonstrated that hydrogen is necessary for the formation of sharp spikes on the surface of a-Si:H thin films irradiated by multiple excimer laser pulses. Hydrogen can also trigger explosive crystallization at the first 15-20 ns, and then crystallized Si on the top will be re-melted in the next tens of nanosecond if the laser energy is high enough. The released pressure by $\mathrm{H}_{2}$ gas bubble explosion at the interface between liquid $\mathrm{Si}$ and solid 
Si will drive the liquid $\mathrm{Si}$ to move up and form as spikes after solidification. Subsequently, the spikes are grown by VLS mechanism until they reach a certain height $(\sim 2 \mu \mathrm{m})$ and the crystallinity also saturates at this point because no more amorphous material underneath the crystallized layer can be melted. The large-grain polycrystallites are located on the spikes and fine-grain ones are distributed underneath, with open cavities caused by hydrogen explosion at the interface. Furthermore, a step-by-step process is taken in order to retain hydrogen as much as possible after processing. It is shown that the residual hydrogen concentration depends on the final fluence, the increment and the number of pulses, and a maximum hydrogen concentration of $3.25 \%$ is obtained at a final fluence of $0.3 \mathrm{~J} / \mathrm{cm}^{2}$ with steps of $0.03 \mathrm{~J} / \mathrm{cm}^{2}$ and 100 pulses for each step. Finally, both single-step and step-by-step laser-processed samples exhibit a similar absorption capability due to the similar surface geometries obtained after different laser processes. It can be concluded that excimer laser step-by-step processing of a$\mathrm{Si}: \mathrm{H}$ thin films may allow for more efficient and stable solar cells.

Acknowledgements Research carried out in part at the Center for Functional Nanomaterials, Brookhaven National Laboratory, which is supported by the U.S. Department of Energy, Office of Basic Energy Sciences, under Contract No. DE-AC02-98CH10886. The use of material characterization equipment at Materials Research Science and Engineering Center, Columbia University, is gratefully acknowledged. The authors also would like to thank Prof. Vijay Modi, Department of Mechanical Engineering, Columbia University, and Prof. Jeffrey M. Gordon, Department of Solar Energy and Environmental Physics, BenGurion University of the Negev, for the helpful discussions and suggestions.

\section{References}

1. A. Luque, S. Hegedus, Handbook of Photovoltaic Science and Engineering (Wiley, Chichester, 2003)

2. K.L. Chopra, P.D. Paulson, V. Dutta, Prog. Photovolt. 12, 69 (2004)

3. L.L. Kazmerski, J. Electron Spectrosc. Relat. Phenom. 150, 105 (2006)

4. R.W. Miles, K.M. Hynes, I. Forbes, Prog. Cryst. Growth Charact. Mater. 51, 1 (2005)
5. D.L. Staebler, C.R. Wronski, J. Appl. Phys. 51 (1980)

6. K.S. Martirosyan, A.S. Hovhannisyan, V.M. Arouiounian, Phys. Status Solidi C 4, 2103 (2007)

7. J.D. Hylton, A.R. Burgers, W.C. Sinke, J. Electrochem. Soc. 151, G408 (2004)

8. C.H. Crouch, J.E. Carey, J.M. Warrender, M.J. Aziz, E. Mazur, F.Y. Genin, Appl. Phys. Lett. 84, 1850 (2004)

9. B.K. Nayak, M.C. Gupta, Appl. Phys. A, Mater. Sci. Process. 89, 663 (2007)

10. K. Yamamoto, A. Nakajima, Y. Masashi, T. Sawada, S. Fukuda, T. Suezaki, M. Ichikawa, Y. Koi, M. Goto, T. Meguro, T. Matsuda, M. Kondo, T. Sasaki, Y. Tawada, Sol. Energy 77, 939 (2004)

11. B.R. Tull, J.E. Carey, E. Mazur, J.P. McDonald, S.M. Yalisove, Mater. Res. Soc. Bull. 31, 626 (2006)

12. D.H. Lowndes, J.D. Fowlkes, A.J. Pedraza, Appl. Surf. Sci. 154, 647 (2000)

13. H. Wang, P. Kongsuwan, G. Satoh, Y.L. Yao, Int. J. Adv. Manuf. Technol. (accepted)

14. H. Wang, P. Kongsuwan, G. Satoh, Y.L. Yao, J. Manuf. Sci. Eng. (under review)

15. J. Im, H. Kim, M. Thompson, Appl. Phys. Lett. 63, 1969 (1993)

16. M. Miyasaka, J. Stoemenos, J. Appl. Phys. 86, 5556 (1999)

17. E. Mathe, A. Naudon, M. Elliq, E. Fogarassy, S. Unamuno, Appl. Surf. Sci. 54, 392 (1992)

18. H. Dehghanpour, P. Parvin, B. Sajad, S. Nour-Azar, Appl. Surf. Sci. 255, 4664 (2009)

19. A. Polman, S. Roorda, P. Stolk, W. Sinke, J. Cryst. Growth 108, $114(1991)$

20. J. Tsao, P. Peercy, Phys. Rev. Lett. 58, 2782 (1987)

21. S. Roorda, W. Sinke, Appl. Surf. Sci. 36, 588 (1989)

22. A. Goetzberger, J. Knobloch, B. Voss, Crystalline Silicon Solar Cells (Wiley, Chichester, 1998)

23. H.S. Carslaw, J.C. Jaeger, Conduction of Heat in Solids, 2nd edn. (Oxford Univ. Press, London, 1958)

24. S. Unamuno, E. Fogarassy, Appl. Surf. Sci. 36, 1 (1989)

25. C.K. Ong, E.H. Sin, H.S. Tan, J. Opt. Soc. Am. B 3, 812 (1986)

26. K.W. Kolasinski, Curr. Opin. Solid State Mater. Sci. 11, 76 (2007)

27. D. Bäuerle, Laser Processing and Chemistry, 3rd edn. (Springer, Berlin, 2000)

28. C.K. Ong, H.S. Tan, E.H. Sin, Mater. Sci. Eng. 79, 79 (1986)

29. A. Bejan, Heat Transfer (Wiley, New York, 1993)

30. A.M. Prokhorov, V.I. Konov, I. Ursu, N. Mihailescu, Laser Heating of Metals (Taylor \& Francis, London, 1990)

31. T. Schwarz-Selinger, D.G. Cahill, S. Chen, S. Moon, C.P. Griogoropoulos, Phys. Rev. B, Condens. Matter 64, 155323 (2001)

32. A. Heya, T. Serikawa, N. Kawamoto, N. Matsuo, Jpn. J. Appl. Phys. 47, 1853 (2008)

33. Q. Hu, Dynamics of melt-mediated crystallization of amorphous silicon films. Ph.D. dissertation, Columbia University (2010)

34. P. Lengsfeld, N. Nickel, W. Fuhs, Appl. Phys. Lett. 76, 1680 (2000) 